\author{
Sugapriya Dhanasekaran* \\ Department of Medical Laboratory Sciences \\ (Hematology), College of Applied Medical Sciences, \\ Prince Sattam Bin Abdullaziz University, Wadi-Ad \\ Dawaser Campus, Riyadh Province, Kingdom of \\ Saudi Arabia \\ Dates: Received: 27 July, 2015; Accepted: 19 \\ August, 2015; Published: 20 August, 2015 \\ *Corresponding author: Sugapriya Dhanasekaran, \\ Department of Medical Laboratory Sciences \\ (Hematology), College of Applied Medical Sciences, \\ Prince Sattam Bin Abdullaziz University, Wadi- \\ Ad Dawaser Campus, Riyadh Province, Kingdom \\ of Saudi Arabia. Tel: 00966-532094216; E-mail: \\ sughaphd@yahoo.com \\ www.peertechz.com \\ ISSN: 2455-8591
}

Keywords: Targeted delivery system

nanomedicines; drug targeting; smart drug;

nanocarriers/vehicles

\section{Review Article \\ SMART Drug Based Targeted Delivery: A New Paradigm for Nanomedicine Strategies}

\begin{abstract}
Introduction: Targeted drug delivery systems are nanoscale drug carrier molecules designed for improving the communication of cellular and molecular components and biodistribution of tumour targeted drug (chemo) therapeutics. Nanomaterials are generally clusters of molecules, atoms and molecular fragments into extremely small size particles $(1-100 \mathrm{~nm})$ in nature. Nanomaterials engineered as self-assembled biodegradable particles were used for targeted drug delivery system. Nanocarriers/ particles should be- capable of transporting high doses of chemotherapeutic drugs/nanomedicines into the targeted tumor cells without disturbing the normal healthy cells. It is also used for construction of novel targeted drug delivery system and future application in nanovaccination and nanotechnology.

Conclusion: Multifunctional smart nanoparticles or carries hold out the possibility of effective drug targeted therapeutics in molecular and cellular levels at the earliest stage. Here, we briefly discuss the significance of targeting strategies and drug delivery system and outline the current approaches and future directions in the improvement of tumor targeting nanomedicines.
\end{abstract}

\section{Introduction}

Nanotechnology has led to a connection of various fields including applied physics, chemistry, optics, biology, computational modelling and analysis, pharmaceuticals and material science. Nanoscales construction provides the ability to analyse and manipulate the stimulation, target the behaviour of biological system or predict the structure by molecular modelling and computational science [1]. Side effects should be avoided by early diagnosis and effective treatment by using nanomedicines [2]. Nanotechnology in health-care research can positively result in enormous health benefits [3] collectively increasing research funding from all over the world [1]. Impacts from improvements progressing from nanotechnology developments over the next 20 years have been assessed to be nearly $\$ 1$ trillion by studies carried out at the International Science Foundation [3]. Nanodevices or sensors and nanomaterials which are used for reparation, monitoring, construction and control of human biological system at cellular and molecular level were known as nanomedicines [3]. Administration of drug by transdermal, oral, intravenous and trans epithelial delivery system is fulfilled using nanocarrier as vehicles. Nanocarriers are present in different forms including polymeric, metal, liposomes, carbon, magnetic and biological particles [4-10] which have a huge biomedical application on drug delivery systems.

Although few products are awaiting for clinical trials, but still nanomedicines are found to be specially promising application and at present used in various fields of diagnosis of diseases, molecular imaging, delivery of chemotherapeutic drug to its targeted tumor sites. Nanomedicines play a significant role by enhancing the efficacy of therapeutic index and safety measurements of new targeted therapeutics even at low dosage levels [9]. Nanocarriers structures which holds conjugated drugs, encapsulated, adsorbed, dispersed and imaging agents. Nanovehicles including nanoemulsions liposomes, micelles, nanoparticulate system (polymer, carbon, drug nanoparticles, ceramic-based, biological, metal, and magnetic) and dendrimers are used for imaging and targeted drug delivery [9]. Freitas Jr. [4] and Flynn and Wei [10] reported the importance of commercialization of nanomedicine including gated nanosieves, immunoisolation, ultrafast DNA sequencing, nanoshells, fullerenebased pharmaceuticals, single-virus detectors, tecto-dendrimers, biologic robots and radio-controlled biomolecules [4]. This review deals with the practical application of nanomedicines and provides an overview of approaches in targeted drug delivery system.

\section{Discussion}

\section{Ideal design for smart drug (Nanomedicine)}

Smart drug which are ideally designed to satisfy the requirements will pave the way for targeted drug delivery. These requirements includes: a) high stability (longevity) and not easily degraded or cleared by the reticuloendothelial system (RES) during blood circulation, b) accumulation of high concentration of drug at the tumor targeted sites or area, c) effective intracellular drug delivery at the tumour targeted area or site that matches drug pharmacodynamics of kinetics and spatial control and d) tolerability. The blood circulation stability and self-life of the drug (size, morphology, low degree of lipid hydrolysis and drug retention) are influenced by the physical and chemical nature of the encapsulated drug and encapsulation techniques. The design of tumor drug targeted delivery system based on: passive drug targeted delivery as a significance of enhanced permeability and retention effect (EPR) (without ligand targets and active drug release mechanisms) [11,12], targeted drug release based on recognition of receptor molecules [13-16], triggered drug release or stimuli - responsive release ( $\mathrm{pH}$, redox potential, temperature, light intensity, ROS response magnetic field, hypoxia response, glucose 
and concentration of electrolytes) to localize the drug-nanocarrier to the determined targeted site [17-20].

\section{Physical and chemical interaction of nanocarrier surface with drug}

Tumor targeted drug should accumulate inside and/or outside of the nanocarriers by interaction with convalent and non-covalent linkage [21]. Encapsulation of targeted drug to nanocarrier is possible during the production of nanocarrier or after the carrier formation. Charging of therapeutic drugs is based on its solubility in the nanocarrier matrix, interaction of drug nanocarrier, the molecular mass of the therapeutic agent and the presence of functional group on a nanocarrier surface [22]. Nanocarrier molecules should hold high purity and chemical resistant nature which might play an important key role to control the targeted drug delivery. Nanocarriers are frequently altered (functionalization) before the encapsulation of targeted drugs [23]. Bioconjugation of polymer with therapeutic drugs leads to enhanced circulation time, minimal toxicity, biodegradation, controlled mechanical properties and well-organised drug release in the targeted sites and their characteristic nature might generate the barrier between cancer cells and nanocarriers [24-28]. Interaction of internal surface of the nanocarrier with entrapped drug molecules are protected beside early activation and biodegradation process therefore interaction of targeted drug with normal healthy cells is impossible [29,30]. Interaction of external surface of the nanocarrier with drug molecules is based on physical and chemical properties, bioconjugation and high surface area of nanocarrier molecules. Therapeutic drug molecule is interacting/bonding with functional groups of nanocarrier by covalently link to form amide, ester or acetyl- hydrazone and disulfide groups. Drugs with aromatic or plane structure can be adsorbed on the surface of nanocarrier by $\pi-\pi$ interactions [31]. Furthermore, drug molecules accumulated on the external surface of the nanocarriers by electrostatic interactions (physical adsorption) [32].

\section{Bioconjugation between nanocarrier and targeted ligand}

Linkage between the targeted ligands with reactive groups of the nanocarriers surface can be categorised into covalent and non-covalent conjugation [33]. Common covalent linkage involve conjugation of two primary amines, two thiol groups, maleimide and thiol, a carboxylic acid and primary amine, hydrazide and aldehyde and a primary amine and aldehyde. Amide bond formation takes place in two stages: 1) triggering of carboxylic group on the surface of nanocarrier molecules, 2) activation of primary amine forming amide bond with nanocarrier molecules that enhance the activity of the applied amine forming amide bond with nanocarrier molecules. This methodology was discriminative and very effective on tumor targeted gene therapy. Thioether bond formed through the reaction between $\mathrm{C} 1$ carbon of maleimide and thiol group that is attached to the $R_{2}$ nanocarrier are stable within 24 hours in human serum even in the existence of reducing agent (e.g DTT) [33-35]. This reaction runs rapidly in aqueous solution as well as at room temperature. Inappropriately, the selectivity towards the thioether group formation is fairly low in aqueous solution, owing to side reactions such as formation of disulfides or intermolecular rearrangement. The thioether bond assures a longer duration for time distribution of drug and high discrimination of targeted drug delivery [34,36]. Alternative way is the coupling of thiol groups of the nanocarrier molecules with fragment of maleimide from the ligands these could be triggered via carboxylic acid or amines groups. This thoether and amide bonding are important in forming bioconjugation of nanocarrier-ligand system [36]. Conjugation of two thiol groups (originates from ligands and nanocarrier molecules) results in the formation of disulfide bond. Formation of disulfide bond between monoclonal antibody antiMy9 and liposomes acts strongly against HL-60 leukemic cells [37]. Bioconjugation of nanocarrier surface to the hydrazide group results in aldehyde groups of the ligands [33,34]. Basically ligands do not possess aldehyde structure but form through oxidation of hydroxyl groups. Conjugation of liposomes with antibodies C225 form acetylhydrazone group showed enhanced production of immunoliposome antibody in in vivo [38]. Advantage of this technique characterized by high immunological expression, prolonged distribution in the blood and can also easily control the immunoliposome synthesis. The cycloaddition reaction between a dienophile and a diene is called Diels-Alder (DA) reaction results in bicyclic compound formation. Coupling of nanocarriers and ligands enhances the yield (nearly to $100 \%$ ) and can be synthesed easily under mild conditions [33]. DA reaction on ligands and nanocarrier linkages causes specific bond formation between the cancer cells and the ligand molecules [36]. Non-covalent bonding arranged by physical interaction of targeted ligands to the nanocarrier surface has an advantage owing to avoiding of severe destruction of therapeutic agents. Weak bonding, low control of reaction and binding of ligands in the undesired orientation are the weakness of the non-covalent interaction [39].

\section{Endocytosis of nanomedicines}

Drug targeted strategy are being validated to address important requirements for targeted therapy, drug delivery system and improved diagnosis. Tumor targeted drug delivery could be classified into carrier and non-carrier based therapy [40]. Carrier based drug therapies comprise, polymeric based drug carriers (polymeric nanoparticles [41], polymeric micelles [42], polymer-drug bioconjugates [43], dendrimers [44]), lipid based drug carriers (liposomes [45]), viral nanocarriers [46], inorganic nanocarriers (quantum dots [47], silica nanoparticles [48], carbon nanotubes [49]) and these nanomaterials with drug collectively called nanomedicine delivered the drug molecules, recombinant DNA and proteins to the targeted tumor cells/tissues/organs. Nanomedicines interact with surface of the targeted tumor cells and undertake intracellular signalling that determine the location (cytoplasm, nuclease) inside the targeted cells and improve the drug accumulation and acquire efficacy of targeted drug delivery [50]. The targeted drug should be stable in the blood circulation enhances the interaction with exterior of the plasma membrane which pave the way to targeted cells named as endocytosis [51,52]. Designed nanocarrier conjugated drug molecules enter into the tumor cells via endocytosis. The specific designed process of endocytosis consist of; a) particles recognition by opsonization in the blood circulation (complement components (C3, C4, C5); absorption of proteins (immunoglobulins; IgG, IgM); and blood serum protein (albumin, fibronectin); b) attachment of opsonized particles into the membrane through receptor present on the targeted surface; c) ingestion of targeted drug by the cell; d) the mature phagosome fused with lysosome and form enzyme-rich phagolysosomes where 
the nanomedicines prone to degradation and show its therapeutic indices $[52,53]$. Recent research proposed nanomedicine transport to the cell membranes based on essential characteristics nature of the nanocarrier (shape, size, hydrophobicity and surface charge) specific interaction between targeted ligand and cell membranes are designed as optimal targeted drug delivery system.

\section{Tumor targeted therapy}

Understanding of molecular mechanism important to tumor progression, formation, angiogenesis, invasion and metastasis clear and pave the way for drug development for targeted drug therapy based on cellular and molecular mechanism. Targeted drug alter the cell signalling pathways and molecular mechanisms (gene function) involved in the pathogenesis of diseases (cancer, parkinson etc...) offer an improved therapeutic value over conventional drugs. Tumor targeted therapy must satisfy few demands. Drug should be delivered to the malignant cells with minimum loss in the concentration and should selectively destroy the targeted tumor cells. Active form of drug release must be controlled simultaneously and minimal doses of therapeutic agents should be administrated for targeted drug therapy than the conventional therapy [54].

Targeted drug therapy also diminishes the possible side effects [36] owing the delivery of targeted drug molecules inside the cells and nanocarriers outside via the passive targeting mechanisms. The conventional therapy is linked with a drug aggregation process (form of carrier-drug) inside the malignant cells via EPR $[55,56]$ thus, drug reaches based on the abnormal structure of the blood vessels near malignant cells [57]. Methotrexate [58], doxorubicin [57], paclitaxel [59], gemcitabine [60] and hexamethylmelamine [61] are most popular chemotherapeutic drugs used in conventional treatment. In tumor targeted therapy system ligands linked to the nanocarriers of therapeutic drugs facilitates the conjugation with specific receptor of the malignant cells. Thus, tumor targeted therapy supports the overexpression of tumor cell receptors have affinity of ligand towards receptor molecules $[36,57,62]$ and delivery of therapeutic agents to the most resistant malignant cells and enhanced the prolonged circulation inside the tumor cells. This application provides high concentration of therapeutic drug inside the tumor cells and targeted drug cannot be released back to the blood circulation [55].

\section{Importance of nanoparticles as targeted drug delivery system}

The therapeutic value of drugs presently being used could be improved efficiently by delivering the drugs into the biological targets via nanoparticles. Drugs failed under the clinical trials might also be reconsidered using nanotechnological methods [63,64]. Drug concentration must be sufficient in the body to permit for an effective dose at the targeted tumor area. The targeted malignant cells must be strongly suppressed by its signalling mechanism essential for its cell survival with high toxicity towards the cancer or positive therapeutic strategies for nanomedicines. Using nanoparticles as carrier molecules a variety of targeted drugs including proteins, biological molecules, vaccines (viral and nonviral vectors), hydrophobic and hydrophilic drugs has been delivered [3]. Hence, eye-ball is a closed organ, an aptamer, antisense oligonucleotide for cytomegalovirus retinitis or a small interfering RNA ( $\mathrm{Si}$ RNA) for age-related macular degeneration (AMD) being investigated in human eye [65]. Architecture of the nanostructured molecules are promising drug carrier that will assist the novel drug compounds towards the targets and has effects on

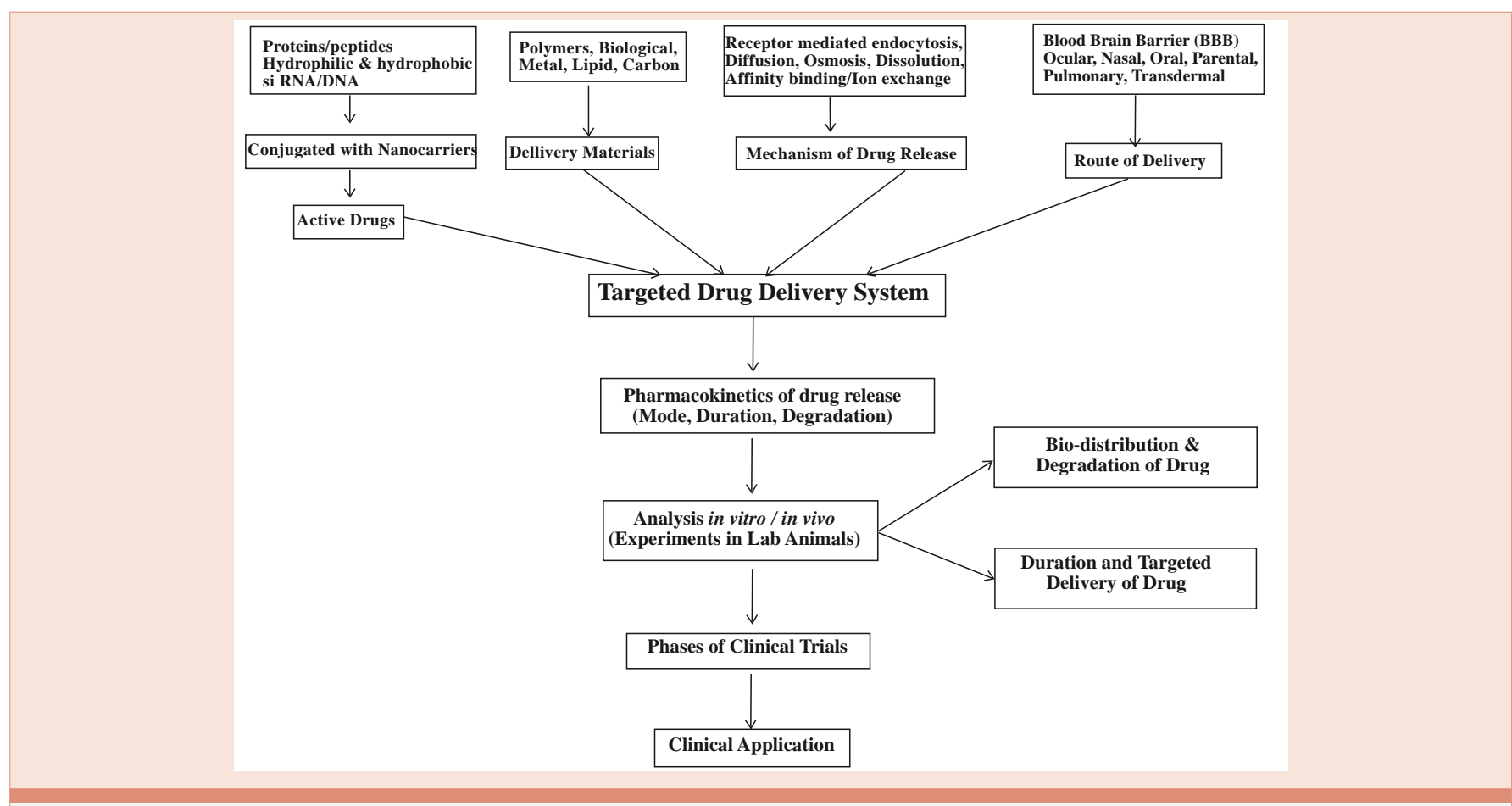

Figure 1: Overview of targeted drug delivery system development from bench to bed. 
continuous drug discharge and capable of intracellular entry. Mostly nanoparticles $[66,67]$ possess an advantages than microparticles that include: intravenous delivery, site-specific drug targeting for various diseases (human immunodeficiency virus infection, cancer, and central nervous system disorders), highly exploited for controlled drug release, prolonged circulation in the blood, facilitate extravasation and passive targeting, avoid opsonization owing to particle size less than $100 \mathrm{~nm}$ and less concentration with prolonged action as well as enhancing the targeting of the drug to specific tumor sites [68]. Targeted drugs can also be administrated via transmucosal, pulmonary, transdermal and implantation delivery other than injection and oral routes.

\section{Future Challenges and Direction}

Application of nanomedicines and improvements of novel targeted drug delivery system play a significant role in tumor therapy. Nanocarriers minimize the enzymatic degradation of therapeutic agent and include consequently improve its activity. Furthermore, it enhances solubility, long-time bio-distribution of drug in the blood circulation and novel system break of biological barriers (blood brain) that improve the therapeutic drug delivered to the targeted tissues/cells/organs. The targeted ligand nanocarrier molecules bioconjugated with drug must meets all basic requirements of safe and effective tumor therapy which include sufficient concentration, effective dosage, high cytotoxicity of therapeutic agents and aggregation of drug molecules on the external and internal surface of the nanocarriers might improves targeted drug delivery (Figure 1). Smart drug nanoparticles must be able to sense the exact alteration in their surroundings to target drug delivery. In addition to targeted drug delivery, smart drugs have application in regenerative medicines (selfregulating scaffolds for cell proliferation or infiltration or injectable systems for cell delivery) and tissue engineering. Development and synthesis of new polymeric based nanoparticles should be expected to be progressively elaborate and versatile nanocarriers of smart drug delivery for implementation in the future. Next generation of polymeric nanocarriers based drug delivery system with hormones, genes, growth factors, peptides, antibodies, etc... may enhance the efficacy and minimize side-effects. Therefore, improvement of multifunctional "smart" drug and nanocarriers pave the way for identification of cancer cells, drugs delivered to the targeted sites, real-time assessment of surgical and therapeutic efficacy, enhanced therapeutic index of multidrug resistance diseases and monitor the intracellular alteration to prevent precancerous cells from becoming malignant. Furthermore, in the future, merging expertise in the drug targeted delivery with technological improvements in nanomedicine should focus on 'bench to bed-side' practises to reduce delay to therapeutic stages.

\section{References}

1. Freitas Jr RA (2005) What is nanomedicine? Nanomedicine 1: 2-9.

2. Mishra B, Patel BB, Tiwari S (2010) Colloidal nanocarriers: a review on formulation technology, types and applications toward targeted drug delivery. Nanomedicine 6: 9-24.

3. Sahoo SK, Parveen S, Panda JJ (2007) The present and future of nanotechnology in human health care. Nanomedicine 3: 20-31.

4. Kostarelos $\mathrm{K}$ (2006) The emergence of nanomedicine: a field in the making
Nanomed 1: 1-3.

5. Wang C, Ravi S, Martinez GV, Chinnasamy V, Raulji P, et al. (2012) Dualpurpose magnetic micelles for MRI and gene delivery. J Control Release 163: 82-92.

6. Mendes RG, Bachmatiuk A, B"uchner B, Cuniberti G, R"ummeli MH (2013) Carbon nanostructures as multi-functional drug delivery platforms. J Mat Chem B 1: 401-428.

7. Wei C (2005) The valuable and significant role of Nanomedicine. Nanomed Nanotech Biol Med 1: 285.

8. Koo OM, Rubinstein I, Onyuksel H (2005) Role of nanotechnology in targeted drug delivery and imaging: a concise review. Nanomed 1: 193-212.

9. Cho KJ, Wang X, Nie SM, Chen Z, Shin DM (2008) Therapeutic Nanoparticles for Drug Delivery in Cancer. Clin Cancer Res 14: 1310-1316.

10. Flynn T, Wei C (2005) The pathway to commercialization for nanomedicine, Nanomedicine 1: 47-51

11. Maeda H (2001) The enhanced permeability and retention (EPR) effect in tumor vasculature: the key role of tumor-selective macromolecular drug targeting. Adv Enzyme Regul 41: 189-207.

12. Maeda H, Wu J, Sawa T, Matsumura Y, Hori K (2000) Tumor vascular permeability and the EPR effect in macromolecular therapeutics: a review. J Control Release 65: 271-284.

13. Jaracz S, Chen J, Kuznetsova LV, Ojima L (2005) Recent advances in tumortargeting anticancer drug conjugates. Bioorg Med Chem 13: 5043-5054.

14. Sofou S, Sgouros G (2008) Antibody-targeted liposomes in cancer therapy and imaging. Expert Opin Drug Deliv 5: 189- 204.

15. Kaasgaard T, Andresen TL (2010) Liposomal cancer therapy: exploiting tumor characteristics. Expert Opin Drug Deliv 7: 225-243.

16. Allen TM (2002) Ligand-targeted therapeutics in anticancer therapy. Nat Rev Cancer 2: 750-763.

17. Drummond DC, Zignani M, Leroux J (2000) Current status of $\mathrm{pH}$ sensitive liposomes in drug delivery. Prog Lipid Res 39: 409-460.

18. Andresen TL, Jensen SS, Jorgensen K (2005) Advanced strategies in liposomal cancer therapy: problems and prospects of active and tumor specific drug release. Prog Lipid Res 44: 68-97.

19. Hatakeyama H, Akita H, Kogure K, Oishi M, Nagasaki Y, et al. (2007) Development of a novel systemic gene delivery system for cancer therapy with a tumor-specific cleavable PEG-lipid. Gene Ther 14: 68-77.

20. Sawant RM, Hurley JP, Salmaso S, Kale A, Tolcheva E, et al. (2006) SMART drug delivery systems: Double-targeted $\mathrm{pH}$-responsive pharmaceutical nanocarriers. Bioconjugate Chem 17: 943-949.

21. Peer D, Karp JM, Hong S, Farokhzad OC, Margalit R, et al. (2007) Nanocarriers as an emerging platform for cancer therapy. Nat Nanotech 2: 751-760.

22. Wong BS, Yoong SL, Jagusiak A, Panczyk T, Ho HK, et al. (2013) Carbon nanotubes for delivery of small molecule drugs. Adv Drug Deli Rev 65:19642015.

23. Sinha R, Kim GJ, Nie S, Shin SM (2006) Nanotechnology in cancer therapeutics: bioconjugated nanoparticles for drug delivery. Mol Cancer Thera 5: 1909-1917.

24. Torchilin V (2009) Multifunctional and stimuli-sensitive pharmaceutical nanocarriers. Eur J Pharma Biopharma 71: 431-444.

25. Khan DR (2010) The use of nanocarriers for drug delivery in cancer therapy. J Cancer Sci Ther 2: 58-62.

26. Yoo HS, Lee KH, Oh JE, Park TG (2000) Invitro and invivo anti-tumor activities of nanoparticles based on doxorubicin- PLGA conjugates. $\mathrm{J}$ of Control Release 68: 419-431. 
27. Yoo HS, Oh JE, Lee KH, Park TG (1999) Biodegradable nanoparticles containing doxorubicin-PLGA conjugate for sustained release. Pharma Res 16: $1114-1118$.

28. Moore TL, Pitzer JE, Podila R, Wang X, LewisRL, et al. (2013) Multifunctional polymer-coated carbon nanotubes for safe drug delivery. Part Part Sys Charac 30: 365-373.

29. Vashist SK, Zheng D, Al-Rubeaan K, Luong JHT, Sheu FS (2011) Advances in carbon nanotube based electrochemical sensors for bioanalytical applications. Biotech Adv 29: 169-188.

30. Marega R, Bonifazi D (2014) Filling carbon nanotubes for nanobiotechnological applications. N J Chem 38: 22-27.

31. Liu Z, Robinson JT, Tabakman SM, Yang K, Dai H (2011) Carbon materials for drug delivery and cancer therapy. Mat Tod 14: 316-323.

32. Niu L, Meng L, Lu Q (2013) Folate-conjugated PEG on single walled carbon nanotubes for targeting delivery of doxorubicin to cancer cells. Macromolecular Bioscience 13: 735-744.

33. Hermanson GH (2008) Bioconjugate Techniques, Academic Press, San Diego, Calif, USA 200-274.

34. Nobs L, Buchegger F, Gurny R, Allémann E (2004) Current methods for attaching targeting ligands to liposomes and nanoparticles. J Pharm Sci 93 1980-1992.

35. Martin FJ, Papahadjopoulos D (1982) Irreversible coupling of immunoglobulin fragments to preformed vesicles. An improved method for liposome targeting J Biol Chem257: 286-288.

36. Liu Y, Miyoshi H, Nakamura M (2007) Nanomedicine for drug delivery and imaging: a promising avenue for cancer therapy and diagnosis using targeted functional nanoparticles. Int J Cancer 120: 2527-2537.

37. Sudhan Shaik M1, Kanikkannan N, Singh M (2001) Conjugation of antiMy9 antibody to stealth monensin liposomes and the effect of conjugated liposomes on the cytotoxicity of immunotoxin. J Control Release 76: 285-295.

38. Harding JA, Engbers CM, Newman MS, Goldstein NI, Zalipsky S (1997) Immunogenicity and pharmacokinetic attributes of poly (ethylene glycol)grafted immunoliposomes. Biochim Biophys Acta 1327: 181-192.

39. Koo OM, Rubinstein I, Onyuksel H (2005) Role of nanotechnology in targeted drug delivery and imaging: a concise review. Nanomedicine 1: 193-212.

40. Houston TA (2007) Painting the target around the arrow: two-step prodrug therapies from a carbohydrate chemist's perspective. Curr Drug Deliv 4: 264 268.

41. Nyman DW, Campbell KJ, Hersh E, Long K, Richardson K. et al. (2005) Phase $\mathrm{I}$ and pharmacokinetics trial of $\mathrm{ABI}-007$, a novel nanoparticle formulation of paclitaxel in patients with advanced nonhematologic malignancies. J Clin Oncol 23: 7785-7793.

42. Nasongkla N, Bey E, Ren J, Ai H, Khemtong C, et al. (2006) Multifunctional polymeric micelles as cancer-targeted, MRI ultrasensitive drug delivery systems. Nano Lett 6: 2427-2430.

43. Bhatt R, de Vries P, Tulinsky J, Bellamy G, Baker B, et al. (2003)Synthesis and in vivo antitumor activity of poly (L-glutamic acid) conjugates of 20 (S)camptothecin. J Med Chem 46: 190-193.

44. Svenson S, Tomalia DA (2005) Dendrimers in biomedical applications reflections on the field. Adv Drug Deliv Rev 57: 2106-2129.

45. Markman M (2006) Pegylated liposomal doxorubicin in the treatment of cancers of the breast and ovary. Expert Opin Pharmacother 7: 1469-1474.

46. Manchester M, Singh P (2006) Virus-based nanoparticles (VNPs): platform technologies for diagnostic imaging. Adv. Drug Deliv. Rev 58: 1505-1522.

47. Tasciotti E, Liu X, Bhavane R, Plant K, Leonard AD Price BK, et al. (2008)
Mesoporous silicon particles as a multistage delivery system for imaging and therapeutic applications. Nat Nanotech 3: 151-157.

48. Nurunnabi M, Choi JS, Huh KM, Lee YH (2010) Targeted near-IR QDsloaded micelles for cancer therapy and imaging. Biomat 31: 5436-5444.

49. Bianco A, Kostarelos K, Prato M (2005) Applications of carbon nanotubes in drug delivery. Curr Opin Chem Biol 9: 674- 679

50. Rajendran L, Knolker HJ, Simons K (2010) Subcellular targeting strategies for drug design and delivery. Nat Rev Drug Discov 9: 29-42.

51. Rejman J, Bragonzi A, Conese M (2005) Role of clathrin- and caveolaemediated endocytosis in gene transfer mediated by lipo- and polyplexes. Mol Ther 12: 468-474.

52. Sahay G, Alakhova DY, Kabanov AV (2010) Endocytosis of nanomedicines. J. Control Release 145: 182-195.

53. Hillaireau H, Couvreur P (2009) Nanocarriers' entry into the cell: relevance to drug delivery. Cellul Mol Life Sci 66: 2873-2896.

54. Bertrand N, Wu J, Xu X, Kamaly N, Farokhzad OC (2014) Cance nanotechnology: the impact of passive and active targeting in the era of modern cancer biology. Adv Drug Deli Rev 66: 2-25.

55. Mehra NK, Mishra V, Jain NK (2014) A review of ligand tethered surface engineered carbon nanotubes. Biomat 35: 1267-1283.

56. Torchilin VP (2006) Multifunctional nanocarriers. Adv Drug Deli Rev 58: 1532 1555.

57. Minotti G, Menna P, Salvatorelli E, Cairo G, Gianni L (2004) Anthracyclines: molecular advances and pharmacologic developments in antitumor activity and cardiotoxicity. Pharmacol Rev 56: 185-229.

58. Samor'I C, Ali-Boucetta H, Sainz R (2010) Enhanced anticancer activity of multi-walled carbon nanotube-methotrexate conjugates using cleavable linkers. Chem Comm 46: 1494-1496.

59. Verweij J, Clavel M, Chevalier B (1994) Paclitaxel (Taxol) anddocetaxel (Taxotere): not simply two of a kind. Ann Oncol 5: 495-505.

60. Aryal S, Hu CMJ, Zhang L (2010) Combinatorial drug conjugation enables nanoparticle dual-drug delivery. Small 6: 1442-1448.

61. Ren Y, Pastorin G (2008) Incorporation of hexamethylmelamine inside capped carbon nanotubes. Adv Mat 20: 2031-2036.

62. Sugapriya D, Bijesh KB, Venil NS, Rama SV (2013) Augmented Sensitivity to Methotrexate by Curcumin induced overexpression of Folate Receptor in KG-1 Cells. Biochimie 95: 1567-1573.

63. Cirillo G, Hampel S, Spizzirri UG. Parisi OI, Picci N, et al. (2014) Carbon nanotubes hybrid hydrogels in drug delivery: a perspective review. Bio Med Res Int Article ID 825017: 17

64. Kipp JE (2004) The role of solid nanoparticle technology in the parenteral delivery of poorly water-soluble drugs. Int J Pharm 284:109-122.

65. Hnik P, Boyer DS, Grillone LR, Clement JG, Henry SP, et al. (2009) Antisense oligonucleotide therapy in diabetic retinopathy. J Diabetes Sci Technol 3: 924-930.

66. Zhang M, Yudasaka M (2014) Potential application of nanocarbons as a drug delivery system. Carbon 69: 642-653.

67. Hughes GA (2005) Nanostructure-mediated drug delivery. Nanomed 1: 22 30.

68. Wilson B, Samanta MK, Santhi K, Kumar KPS, Ramasamy M, et al. (2010) Chitosan nanoparticles as a new delivery system for the anti-Alzheimer drug tacrine. Nanomedicine 6: 144-152.

Copyright: (c) 2015 Dhanasekaran S. This is an open-access article distributed under the terms of the Creative Commons Attribution License, which permits unrestricted use, distribution, and reproduction in any medium, provided the original author and source are credited. 